\title{
Tecnologia Digital na formação permanente de profissionais de enfermagem em ambiente hospitalar
}

\author{
Digital Technology in the permanent training of nursing professionals in hospital environment \\ La Tecnología Digital en la formación continuada de los profesionales de la enfermería en un \\ entorno hospitalario
}

Recebido: 28/07/2021 | Revisado: 03/08/2021 | Aceito: 04/08/2021 | Publicado: 09/08/2021

Fernanda Diniz Flores

ORCID: https://orcid.org/0000-0001-8644-9201

Universidade Feevale, Brasil

E-mail: fernandaflores@feevale.br

Débora Nice Ferrari Barbosa

ORCID: https://orcid.org/0000-0001-8107-8675

Universidade Feevale, Brasil

E-mail: deboranice@feevale.br

Marta Rosicler Bez

ORCID: https://orcid.org/0000-0002-5542-8229

Universidade Feevale, Brasil

E-mail: martabez@feevale.br

\begin{abstract}
Resumo
Este artigo apresenta uma revisão sistemática da literatura, analisando como as tecnologias digitais são adotadas na educação permanente dos profissionais da saúde, mais especificamente aos profissionais de enfermagem que atuam em ambiente hospitalar. Para tanto, utilizou-se as bases de artigos IEEE eXplore, ACM Digital, Pubmed, Science Direct, Scopus e Willey. Foi criado um protocolo e inserido na ferramenta Start para seleção dos artigos, através de filtros e cinco fases. Iniciou-se a busca com 738 artigos, que foram descartados de acordo com as fases. Ao final, para a leitura completa e análise, restaram seis artigos, todos internacionais. Percebe-se na leitura completa dos artigos que algumas ferramentas tecnológicas são utilizadas, porém, ainda de forma superficial e não desenvolvidas para este fim. Neste sentido, identifica-se uma lacuna de pesquisa o pouco uso de tecnologias na formação permanente de enfermeiras.
\end{abstract}

Palavras-chave: Formação permanente em enfermagem; Engajamento; Revisão sistemática da literatura; Aprendizagem ao longo da vida; Tecnologia digital.

\begin{abstract}
This article presents a literature systematic review, analyzing how digital technologies are being adopted to help nurses that work in hospitals in the lifelong education perspective. The bases researched were IEEE explore, ACM Digital, Pubmed, Science Direct, Scopus and Willey. The Start tool was used to applied the protocol and to select the articles, through filters and five phases. The search was started with 738 articles, which were discarded according to the phases. Six international articles were select for complete reading and analysis. It was concluded that some technological tools are used in a superficial way and not developed for the purpose of training nurses that work in hospitals. The results identified research's gap in the use of technologies in the nurses permanent training.
\end{abstract}

Keywords: Nursing continuing education; Engagement; Systematic review of literature; Lifelong learning; Digital technology.

\section{Resumen}

Este artículo presenta una revisión sistemática de la literatura, analizando cómo se adoptan las tecnologías digitales para ayudar a la formación continua de los profesionales de la salud, más concretamente de los profesionales de la enfermería que trabajan en los hospitales. Para ello, se utilizaron las bases de datos de artículos IEEE eXplore, ACM Digital, Pubmed, Science Direct, Scopus y Willey. Se creó un protocolo que se insertó en la herramienta Start para la selección de artículos, mediante filtros y cinco fases. La búsqueda comenzó con 738 artículos, que se descartaron según las fases. Al final, para la lectura y el análisis completos, quedaron seis artículos, todos ellos internacionales. La lectura completa de los artículos revela que algunas herramientas tecnológicas se utilizan, sin embargo, todavía de forma superficial y no desarrolladas para este fin. Se puede identificar como una brecha de investigación el poco uso de las tecnologías en la formación continua de las enfermeras.

Palabras clave: Educación continua en enfermería; Implicación; Revisión sistemática de la literatura; Aprendizaje permanente; Tecnología digital. 


\section{Introdução}

A formação permanente faz parte da educação em saúde, constituindo-se de uma prática de ensino-aprendizagem (BRASIL, 2018). A Educação Permanente deve promover, além da capacitação técnica específica necessária dos sujeitos, a aquisição de novos conhecimentos, conceitos e principalmente atitudes, as quais, influenciam a prática. Portanto, podemos dizer que é uma "vertente" intrínseca, uma capacidade a ser desenvolvida, uma competência, "é o aprender constante em todas as relações do sujeito" (Paschoal; Mantovani \& Méier, 2007, p. 480).

Esta contribuição contínua e ao longo da vida coopera com a construção de um sistema de saúde de atualização constante, desta forma promovendo melhorias no atendimento e, por consequência, qualidade do cuidado aos pacientes (Adamy et al., 2018) (Flores, Oliveira, \& Zocche, 2016). A área da saúde é um constante de novas informações e o profissional necessita ter engajamento para alinhar o conhecimento a prática. O resultado dessa junção é a prestação de um cuidado com qualidade, unindo técnica a humanização, que consequentemente influencia na receita da instituição prestadora de cuidados e tratamento de saúde (Almeida, Teston, \& Medeiros, 2019). O uso das tecnologias digitais tem um papel importante neste cenário.

As tecnologias digitais vêm contribuindo para aprendizagens ativas, auxiliando na interação, otimizando o tempo, e a construção do saber. Conforme Bacich e Moran (2017) a tecnologia digital móvel combinada com as metodologias ativas é uma inovação do ensino, ampliando as possiblidades de pesquisa. Ainda os autores, descrevem que as "tecnologias digitais diluem, ampliam e redefinem a troca entre os espaços formais e informais por meio de redes sociais e ambientes abertos de compartilhamento e coautoria" (Bacich \& Moran, 2017, p. 12).

Bes (2017), relata que um dos princípios que incentiva o interesse do aprendiz é o impacto do conteúdo na resolução de problemas do cotidiano, a motivação para aprender é mais intrínseca e está relacionada a autorrealização e reconhecimento. Munhoz (2017) contribui ao dizer que o incentivo deve ser a aprendizagem não apenas a absorção do conteúdo. Percebe-se uma conexão entre a formação profissional permanente e o alcance da tecnologia digital de uma forma social presente no dia a dia. Antes o uso de celulares na prática de ações direcionadas ao trabalho ou ao estudo era tido como objeto de desvio de atenção. Hoje virou um acessório ubíquo ${ }^{1}$, contribuindo como ferramenta de pesquisas rápidas, de troca de informações, interações e compartilhamento de conhecimento, bem como meio de resposta rápida a ações que são informadas, proporcionando feedback em tempo real. Logo, o uso das tecnologias, em especial a móvel, impacta também os processos profissionais, inclusive na área da saúde. Neste tempo atual (pandemia covid-19), firma-se através da repercussão de tamanha preocupação mundial, que a profissão de enfermagem é reconhecida como utilidade social. Em ambiente hospitalar, cada colaborador possui suas individualidades, potencialidades e seus valores motivacionais. Uma boa comunicação implica na descoberta desses elementos e formas de manter a equipe motivada e engajada na qualidade do serviço prestado (Marquis \& Huston, 2005). Neste contexto, a eficiência e a produtividade dos recursos humanos estão associadas a satisfação no trabalho e, este, tem impacto no serviço prestado, na qualidade do cuidado e segurança do paciente, fortalecendo o compromisso com a instituição (Spader, 2019).

Neste sentido, este artigo busca compreender: como as tecnologias digitais são adotadas para auxílio na educação permanente aos profissionais da saúde, mais especificamente aos profissionais de enfermagem que atuam em ambiente hospitalar? Para responder a esta questão, foi realizada uma revisão sistemática da literatura analisando o período de 10 anos de publicações (de 2010 até agosto de 2020). Foram analisadas 6 bases de dados, resultando em 738 artigos. Ao final, como resultado do processo de busca, foram analisados 6 trabalhos. As bases que mais resultaram nesta pesquisa foram PubMed e

${ }^{1}$ Computação ubíqua é um termo usado para descrever a onipresença dos computadores no cotidiano das pessoas (Wunsch, Righi, Costa, \& Oliveira, 2018). 
Science Direct. Ferramentas síncronas e assíncronas surgiram como exemplos de métodos de educação permanente em ambientes hospitalares, fomentando o conhecimento influenciando na prática. Percebe-se, através deste trabalho, um crescente número de publicações do tema.

Este artigo está organizado em cinco seções, além desta introdução. Na seção dois apresenta-se a Metodologia utilizada para estabelecer o processo de busca e análise. Na seção três os Resultados obtidos são apresentados, a partir da análise dos trabalhos selecionados. Na quarta seção é apresentada a Discussão entre os resultados alcançados na revisão da literatura. Finalmente, a seção cinco apresenta as Conclusões do trabalho.

\section{Metodologia}

O trabalho envolveu o processo metodológico sistemático proposto por Petersen, Vakkalanka e Kuzniarz (2015), cuja execução consiste em (1) definir a questão de pesquisa, (2) definir o processo de busca, (3) estabelecer os critérios para o filtro dos resultados, (4) executar as análises e classificar os resultados.

\subsection{Questões de Pesquisa}

A questão de pesquisa que norteia este trabalho é: como as tecnologias digitais são adotadas para auxílio na educação permanente aos profissionais da saúde, mais especificamente aos profissionais de enfermagem que atuam em ambiente hospitalar? Para nortear o processo de estudo foram definidas as questões gerais (QG), Questão Focal (QF) e Questões estatísticas (QE), apresentadas no Quadro 1.

Quadro 1 - Questões de pesquisas.

\begin{tabular}{|c|c|}
\hline QG1 & Quais recursos tecnológicos estão sendo utilizados na formação permanente? \\
\hline QG2 & Qual o foco da interação horizontal entre equipe e gestão? \\
\hline QG3 & $\begin{array}{l}\text { Que indicadores de qualidade de serviço hospitalar permitem mensurar o } \\
\text { engajamento das equipes? }\end{array}$ \\
\hline QF1 & Quais são os conteúdos abordados na formação permanente com tecnologias? \\
\hline QE1 & Qual a distribuição de artigos por região? \\
\hline QE2 & Quais são as tendências em termos de regularidade percebidas? \\
\hline
\end{tabular}

Fonte: Elaborado pelas autoras, com base nos dados da pesquisa (2020).

\subsection{Processo de Pesquisa}

Para atender aos objetivos propostos, foram extraídas palavras chaves, sendo: Tecnologia digital. Enfermagem. Hospital. Educação Permanente. Engajamento. Saúde. A partir destas, gerou-se a string de busca, onde as chaves obrigatórias são "Engajamento" "Hospital", "Aprendizagem ao longo da vida", "Tecnologia digital", "Saúde" e "Enfermagem". Desta forma, a expressão de busca foi dividida em seis conjuntos de interesse. Estes são unificados por conjunções booleanas.

As demais chaves de busca, apresentam variações de singular e plural, palavras derivadas de radical, termos que contemplam o mesmo significado, foram aplicadas de forma a abranger o maior número de resultados. Esta string foi aplicada nos motores de busca definidos para esta revisão; adaptadas para refinar seu conteúdo, e possibilitar um resultado apurado à 
busca. A string de busca aplicada é apresentada a seguir: "engajamento OR engagement" AND "hospital" AND "formação OR formação permanente" AND "tecnologia OR digital OR digital technology" AND "saúde OR Health" AND "enfermagem OR nursing".

Após análise das bases de maior interesse, foram definidas seis a serem utilizadas nesta revisão. Em vistas da interdisciplinaridade, buscou-se artigos em bases com foco na área de tecnologias, educação e saúde. Desta forma, serão utilizadas as bases de dados:

- IEEExplore, que fornece acesso para mais de três milhões de documentos de algumas das publicações mais citadas no mundo em Engenharia Elétrica, Ciência da Computação e Eletrônica (IEEEXPLORE, 2020);

- ACM Digital Library, base de dados bibliográficos com foco na área da computação (ACM DIGITAL LIBRARY, 2020).

- Para a área da saúde, será utilizada a base de dados PubMed. A mesma conta com citações da literatura biomédica do MEDLINE (PUBMED, 2020).

- SCIENCE DIRECT, plataforma para acesso de aproximadamente 2500 revistas científicas e mais de 26000 e-books (SCIENCE DIRECT, 2020);

- SCOPUS, plataforma que oferece um panorama da produção de pesquisas do mundo nas áreas de ciência, tecnologia, medicina, ciências sociais, artes e humanidades (SCOPUS, 2020); e

- plataforma WILEY, que contém conteúdo multidisciplinar, incluindo as áreas de Ciências da Saúde, Biológicas, Exatas e da Terra, Agrárias, Sociais Aplicadas, Humanas, Linguística, Letras e Artes. Para acesso irrestrito às bases, utilizou-se o convênio da universidade onde o trabalho foi desenvolvido.

Após a definição da string de busca, ela foi executada nos motores de buscas já definidos. As referências coletadas destas bases foram registradas na ferramenta StArt (State of the Art through Systematic Reviews) (FABBRI et al., 2016), para auxílio no processo de gerenciamento e catalogação. O processo de pesquisa desenvolveu-se em 5 fases distintas:

- Fase 1: aplicar a string de busca nas bases selecionadas;

- Fase 2: aplicar os critérios de inclusão e exclusão;

- Fase 3: leitura do título, palavras-chave e resumos;

- Fase 4: leitura completa da introdução e conclusão;

- Fase 5: leitura integral dos artigos restantes para responder às perguntas da presente pesquisa.

\subsection{FILTRO DE RESULTADOS}

Os critérios para inclusão (CI) ou exclusão (CE) dos artigos neste estudo precisam obedecer às seguintes características:

(CI1) O artigo deve ter sua publicação entre os anos 2010 a 2020;

(CI2) Ser um artigo científico;

(CI3) O artigo deve estar escrito no idioma inglês ou português;

(CI4) O ambiente de utilização deve ser terciário de atendimento à saúde;

(CI5) A população alvo é da categoria profissional em enfermagem.

(CE1) Serão descartadas teses ou dissertações;

(CE2) Excluídos estudos relacionados a acadêmicos em enfermagem;

(CE3) Serão descartados trabalhos que citam ambientes primários ou secundários;

(CE4) Excluídos artigos que não contemplam o objetivo desta pesquisa. 


\section{Fase 1 e 2- Aplicação da String nas bases e aplicação dos CI e CE}

Inicialmente a string de busca foi lançada nos motores de busca (Fase 1). Na plataforma ACM a string foi aplicada em "todos os campos" para gerar resultados, bem como na plataforma IEEE. Na Scopus, a string foi lançada de modo que pudessem ser encontradas no título do artigo, no resumo, ou nas palavras-chave. Na plataforma da Science Direct a busca sucedeu com os termos em inglês, optado por utilizar apenas artigos que abrangem as disciplinas de medicina e saúde pública; educação; psicologia; ciências da computação e filosofia. Quanto as subdisciplinas, foram contempladas a educação médica; medicina interna; imagem/radiologia; medicina nuclear. Na plataforma Wiley os filtros utilizados foram saúde e cuidados a saúde, bem como publicações em revistas. Todos os termos da string foram buscados no campo "anywhere". Especificamente na plataforma PubMed, para obter os resultados, foram necessárias duas buscas: primeiro aplicação de toda a string (fiel) pelo abstract (retornando 7082 artigos) e pelo título (retornando 458 artigos). Após esta primeira busca, usa-se o "all fields" as duas buscas (builder $A N D$ ), obtendo o resultado utilizado para esta revisão.

Após, aplicou-se os filtros CI1, CI2, CI3, CE1 em todos os motores de busca, e procedeu-se com a importação dos artigos na ferramenta Start. A ferramenta permite remover os artigos duplicados na importação, resultando em um total de 738 artigos. A Tabela 1 demonstra os resultados obtidos em cada motor de busca.

Tabela 1- Resultados para cada base consultada.

\begin{tabular}{c|c} 
Base & Total \\
\hline ACM & 4 \\
\hline IEEE & 43 \\
\hline Scopus & 19 \\
\hline Pubmed & 344 \\
\hline Science Direct & 280 \\
\hline Wiley & 48 \\
\hline Total geral & $\mathbf{7 3 8}$
\end{tabular}

Fonte: Elaborado pelas autoras, com base nos dados da pesquisa (2020).

A seguir, na Figura 1, pode ser observada a representatividade dos motores de busca através de um gráfico dos resultados obtidos. Na sequência, serão apresentados os resultados para cada fase de seleção. 
Figura 1 - Resultados obtidos na fase 1 .

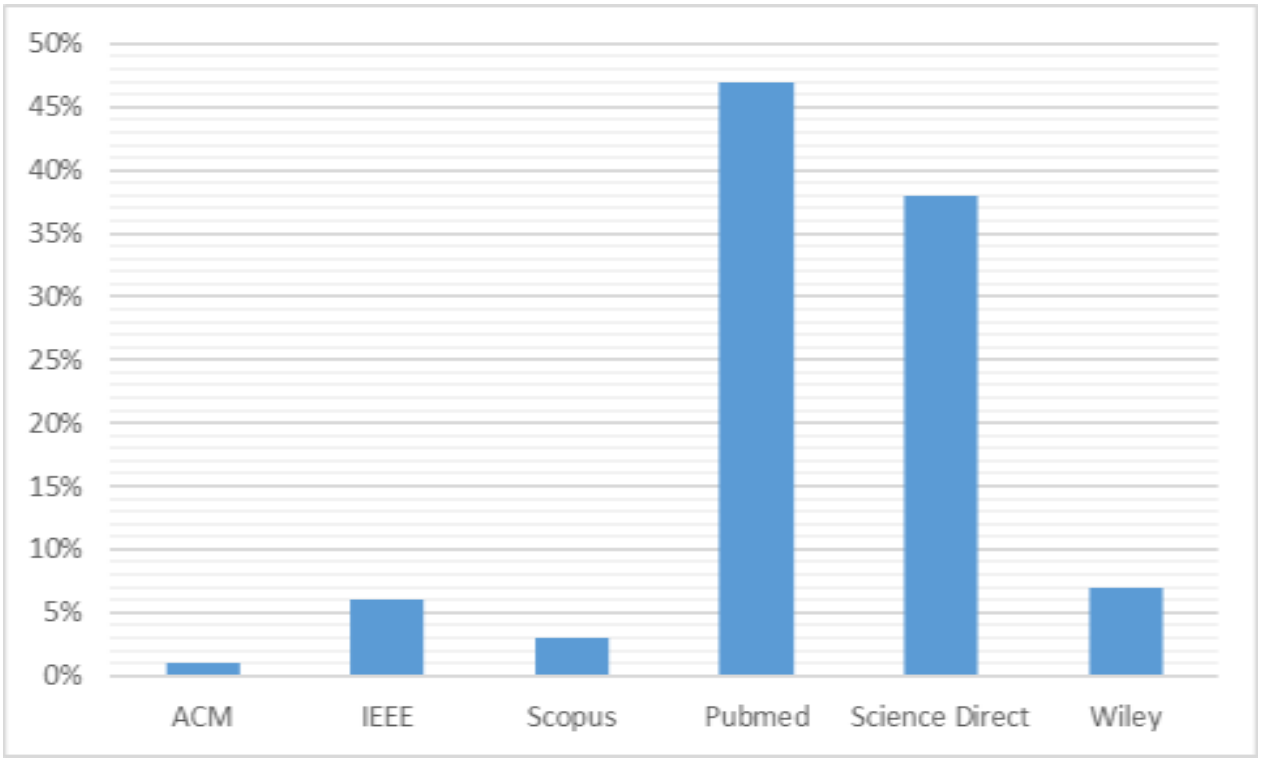

Fonte: Elaborado pelas autoras, com base nos dados da pesquisa (2020).

A seguir, realizou-se o processo de seleção dos artigos já importados na ferramenta Start, aplicando os critérios de inclusão e exclusão do protocolo. Como os motores de busca possibilitam a utilização de filtros avançados, os critérios, CI1 e CI2 foram aplicados anteriormente, ao realizar a consulta, e desta forma não serão validados novamente (fase 2).

A seguir, aplicou-se os demais critérios de inclusão e exclusão e segue-se as demais fases do método.

\section{Fase 3 - Leitura de Títulos, palavras-chave e resumos}

Nesta etapa foram lidos os títulos, as palavras-chave e os resumos, objetivando validar o foco de pesquisa presente nas publicações selecionadas. Foi possível, também, nesta fase, identificar artigos que condizem com o CE3 (não serão utilizados trabalhos que citam ambientes primários ou secundários de atendimento à saúde). A PubMed e Science Direct apresentaram 82 e 2 artigos respectivamente que citam ambientes primários e secundários da saúde. Obteve-se um resultado de 47 publicações aceitas e 621 rejeitadas após análise dos temas.

\section{Fase 4- Leitura da introdução e conclusão}

Nesta fase, procedeu-se a leitura da introdução e conclusão dos artigos aceitos anteriormente, pretendendo vincular ao tema de pesquisa e a busca de tecnologias digitais usadas na formação profissional. Após a leitura da introdução e conclusão de 47 artigos, 6 artigos foram selecionados para a próxima fase. Os artigos aceitos, com seus títulos, respectivos autores e anos de publicação encontram-se no Quadro 2. 
Quadro 2 - Quadro sinóptico - Artigos finais para análise.

\section{TÍTULOS}

FRISCH et al. (2014)

FRENK et al. (2010)

PATA; SANTOS \& BURCHERT (2016)

MATHEW (2014)

MURPHY et al. 2015)
Growing a Professional Network to Over 3000 Members in Less Than 4 Years: of InspireNet, British Columbiaâ€ $\mathbf{T M}_{S}$ Virtual Nursing Health Services

Health professionals for a new century: transforming education to strengthen health systems in an interdependent world

Social recognition provision patterns in professional Q\&A forums in Healthcare and Construction

Using a social networking tool for blended learning in staff training: Sharing experience from practice

Translating research into practice: Evaluation of an e-learning resource for health care professionals to provide nutrition advice and support for cancer survivors

PIMMER et al. (2019)

\section{Facilitating professional mobile learning communities with instant messaging}

Fonte: Elaborado pelas autoras, com base nos dados da pesquisa (2020).

\section{Fase 5 - Leitura completa dos artigos}

Como última etapa de seleção dos artigos, foi realizada a leitura completa dos estudos selecionados e a aplicação das questões de pesquisa, definidas previamente no protocolo. O fluxograma 1 apresenta o processo de seleção do estudo para revisão e os resultados obtidos. A Tabela 2, detalha, em termos numéricos, o processo de inclusão e exclusão dos artigos dentro do contexto de cada base de dados.

\section{Fluxograma 1- Processo de seleção do estudo para revisão}

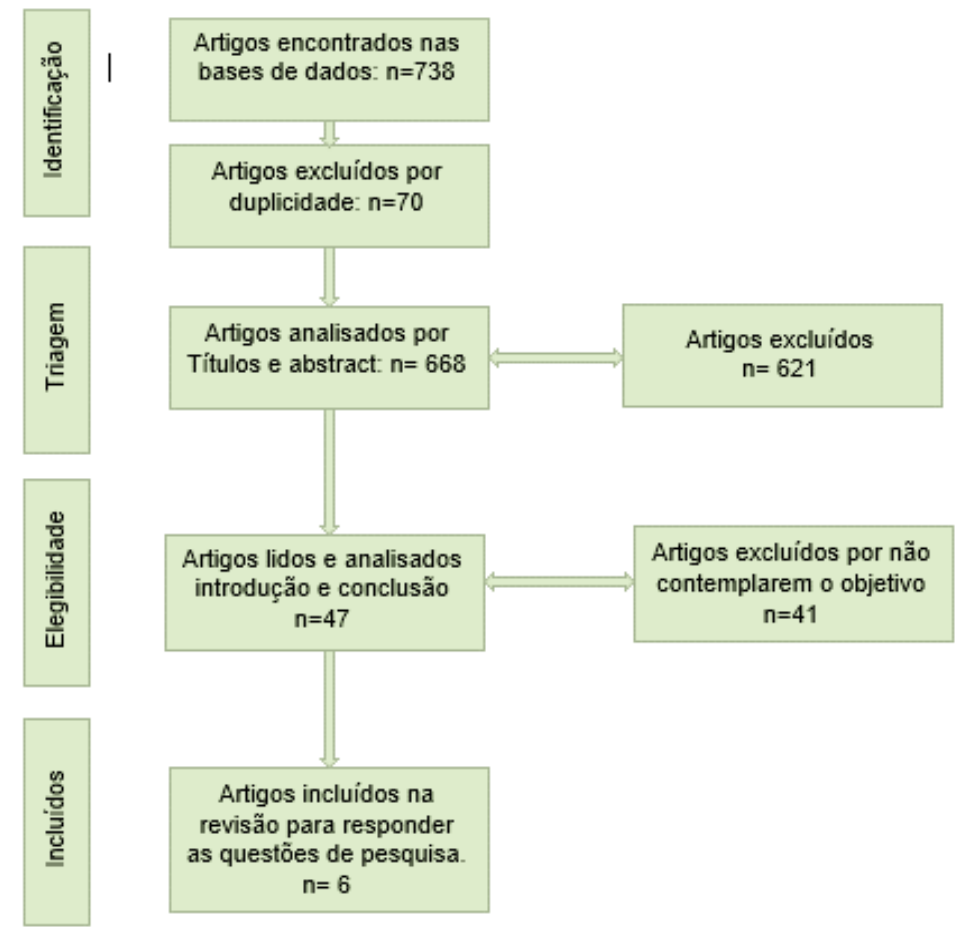

Fonte: Prisma Statement. Adaptado de Moher et al. (2009) 
Todos os artigos selecionados estão de acordo com os critérios de inclusão e objetivo desta revisão. A amostra final constitui-se de 6 artigos, publicados na base de dados PubMed e Science Direct. Dentre os artigos destaca-se 2014 como o ano de maior prevalência, com dois artigos publicados, já os anos de 2010, 2015, 2016 e 2019 tiveram uma publicação. Estes artigos fazem parte da publicação de periódicos de 6 revistas: Nurse Education Today; Computers \& Education; Journal of Medical Internet Research; The Lancet; Computers in Human Behavior; Journal of Neonatal Nursing.

Tabela 2 - Resultados do processo de busca.

\begin{tabular}{|c|c|c|c|c|c|c|c|c|}
\hline & \multirow[b]{2}{*}{ TOTAL } & \multirow[b]{2}{*}{ DUPLICAT. } & \multicolumn{2}{|c|}{ Fase 3} & \multicolumn{3}{|c|}{ Fase 4} & \multirow{2}{*}{$\begin{array}{l}\text { Fase } 5 \\
\text { Aceitos }\end{array}$} \\
\hline & & & Rejeitados & Aceitos & CE2 & CE3 & CE4 & \\
\hline PubMed & 344 & 49 & 283 & 12 & 1 & & 10 & 1 \\
\hline Scopus & 19 & & 19 & & & & & \\
\hline $\mathbf{A C M}$ & 4 & & 3 & 1 & & & 1 & \\
\hline Science Direct & 280 & 7 & 241 & 32 & 3 & & 24 & 5 \\
\hline Wiley & 48 & 13 & 33 & 2 & & & 2 & \\
\hline IEEE & 43 & 1 & 42 & & & & & \\
\hline Total & 738 & 70 & 621 & 47 & 4 & 0 & 37 & 6 \\
\hline
\end{tabular}

Fonte: Elaborado pelas autoras, com base nos dados da pesquisa (2020).

A bases de dados que mais resultaram em pesquisas foram a PubMed, com 344 artigos, seguida da Science Direct com 280 artigos, sendo estas as que contribuíram com maior número de artigos aceitos. Também se percebe através da avaliação da Tabela 3, o número de artigos duplicados em cada base de dados, sendo na PubMed e Wiley as bases de maiores achados de duplicatas, 49 e 13 respectivamente.

Dentre os 47 artigos avaliados para a Fase 4, o CE4 (excluídos artigos que não contemplam o objetivo da pesquisa) é o critério que resulta em uma quantidade menor de artigos selecionados para a fase 5. Ainda, a base de dados IEEE contribui com 43 artigos (Fase 1 e 2), sendo uma duplicata, porém os 42 artigos foram rejeitados logo na Fase 3, por não contemplarem o objetivo da pesquisa. Do mesmo modo, sucede com a base de dados Scopus, porém com o retorno de 19 artigos nas Fases 1 e 2.

Muitos artigos foram descartados logo após a leitura do título, palavras-chave e resumo, devido ao objetivo deste trabalho, resultando, portanto, em 6 artigos para responder as perguntas de pesquisa.

\section{Resultados e Discussão}

\section{QG1- Quais recursos tecnológicos estão sendo utilizados na formação permanente?}

Os recursos tecnológicos foram abordados nos aspectos quanto a educação permanente a profissionais da enfermagem e tecnologia digital. Pode-se observar o uso de plataformas desenvolvidas para este fim, como traz Frisch et al. (2014) com o InspireNet (Serviços e práticas inovadoras de enfermagem informados por rede de pesquisa e avaliação), buscando aumentar a capacidade e o interesse dos enfermeiros pela pesquisa em serviços de saúde em British Columbia (BC), Canadá (Frisch et al., 2014). O artigo de Frenk et al. (2010), traz o exemplo da OpenCourseWare (OCW), uma plataforma de publicação de material 
de curso, sendo caracterizado de aprendizagem transformadora. Já a pesquisa Pata; Santos e Burchert (2016), traz os fóruns de perguntas e respostas. Estudam três plataformas do setor da saúde: Linkedin 'MyHealthSkills' e a plataforma Fórum de QA para enfermeiras clínicas².

Mathew (2014), traz a experiências de enfermeiras ao utilizarem rede social e uma página aberta chamada de "NIG", para compartilhamento de experiências e aprendizagem. Murphy et al. (2015), de forma geral, cita recursos úteis ou informativos (videoclipes, desenhos animados, áreas de reflexão e links) da Web como forma de aprendizagem em um processo de instrução sobre nutrição e cuidados a enfermos de câncer. Pimmer et al. (2019), traz o uso de Mobile Instant Messaging (MIM), o WhatsApp como meio de compartilhamento de conhecimento entre grupos, auxiliando na transição de recém formados na experiência do primeiro emprego (Pimmer et al., 2019)(Pimmer et al., 2019)(PIMMER et al., 2019)(PIMMER et al., 2019)(PIMMER et al., 2019)(PIMMER et al., 2019)(PIMMER et al., 2019)(PIMMER et al., 2019).

\section{QG2 - Qual o foco da interação horizontal entre equipe e gestão?}

A interação horizontal entre equipe e gestão pode ser categorizada de acordo com o foco tecnologia, comunicação e compartilhamento de conhecimento (informações). Este meio de relação auxilia no trabalho conjunto, cada trabalhador contribui com potencialidade e conhecimentos individuais, auxiliando na construção de uma realidade (Marquis \& Huston, 2005). Os autores Pimmer et al. (2019), retratam este ponto ao citar o início da carreira profissional. Esta, é marcada pelo isolamento profissional, altos níveis de estresse e baixa satisfação no trabalho (especialmente nos primeiros 6 a 9 meses no emprego). O uso da tecnologia digital apoia o desenvolvimento profissional, aumenta o compartilhamento do conhecimento e promove a comunicação profissional formal / informal.

Murphy et al. (2015) citam a utilização do equivalente recurso (tecnologia digital) e formação contínua para atingir o mesmo objetivo, realizar orientação, baseada em estudos científicos, sobre a nutrição a pacientes com câncer. Do mesmo modo, Mathew (2014), sinalizou as potencialidades desenvolvidas da equipe da neonatologia através do recurso de aprendizagem implantado.

Os autores, Frisch et al. (2014), Frenk et al. (2010) e Pata; Santos \& Burchert (2016), inferem as relações secundárias originadas a partir do uso de tecnologias digitais, na forma de compartilhamento de conhecimento em sistemas colaborativos.

\section{QG3 - Que indicadores de qualidade de serviço hospitalar permitem mensurar o engajamento das equipes?}

Após a contratação de enfermeiras sem experiência na área de neonatologia, Mathew,(2014) relata a preocupação da instituição e como as tecnologias auxiliaram na formação destes profissionais. Tão logo houve o uso de tecnologia digital como meio de educação continuada, percebeu-se um melhor desempenho, abordagens proativas no atendimento, refletindo na melhora significativa da segurança do paciente.

Pimmer et al. (2019), consideram que o uso do WhatsApp pode aumentar o conhecimento e a conexão profissional. Nos demais artigos, não foi possível perceber de forma clara os elementos relacionados a qualidade de serviço, embora a partir da leitura completa dos trabalhos, pode-se inferir atitudes positivas com potencial para melhorias na base de conhecimento e mudanças na prática profissional de uma forma coletiva.

\section{QF1 - Quais são os conteúdos abordados na formação permanente com tecnologias?}

Pata; Santos \& Burchert (2016), mencionam conteúdos relativos a normas, dinâmica social e materiais, com o objetivo de resolver problemas práticos, neste estudo trouxeram como exemplos os setores da saúde e construção civil.

\footnotetext{
2 (http://www.practicenursing.co.uk/forum/home.aspx)
} 
Já Frisch et al. (2014) abordam conteúdos visando aumentar a capacidade e o interesse dos enfermeiros pela pesquisa em serviços de saúde em British Columbia (BC), Canadá. Os usuários se beneficiaram da rede de várias maneiras, em termos de construção de capacidade de pesquisa, conexão e comunicação com outros profissionais e apoio à sua prática.

Os artigos de Frisch et al. (2014) e Murphy et al. (2015), trouxeram conteúdo específicos relacionados ao setor de prática, a neonatologia e nutrição respectivamente. A pesquisa de Murphy et al. (2015) disponibilizou conteúdos de planejamento de carreira ou segurança no trabalho e conhecimentos clínicos práticos como: o manejo de doenças transmissíveis, avaliação da dor, arrumação da cama ou uso de cateterismo vesical.

\section{QE1 - Qual a distribuição de artigos por países?}

Para identificar os países onde os trabalhos foram desenvolvidos, considerou-se a instituição de origem dos autores dos artigos e referência ao país no texto. No quadro 3 abaixo essa informação consta organizadamente.

\section{Quadro 3- Distribuição de artigos por países}

Artigos País de Origem

Frisch et al. (2014) Canadá - Bristish Columbia

Frenk et al. (2010) Comissão de 20 líderes profissionais e acadêmicos de diversos países, dentre eles: China; Canadá; USA; Paquistão

\begin{tabular}{cl}
\hline Pata et al., (2016) & Estônia- Universidade de Tallinn \\
\hline Mathew, (2014) & Inglaterra- Universidade Londres \\
\hline Murphy et al., (2015) & Inglaterra - Universidade de Bournemouth \\
\hline Pimmer et al., (2019) & Suíça - Universidade de Ciências aplicadas e artes do Noroeste da Suíça \\
\hline
\end{tabular}

Fonte: Elaborado pelas autoras, com base nos dados da pesquisa (2020).

Como demonstrado anteriormente, todos os artigos têm origem internacional, apesar de não haver critérios de inclusão e exclusão a este respeito. Neste sentido, nenhum artigo selecionado é desenvolvido no Brasil.

\section{QE3 - Quais são as tendências em termos de regularidade percebidas?}

Para esta análise, utilizou-se o estudo dos 47 artigos (originados a partir da leitura do título e resumo - na fase 3), deste modo, obtendo uma margem ampla da tendência, levando em consideração que todos eles traziam o tema tecnologia digital, embora 41 foram descartados por não contemplarem especificamente o objetivo da pesquisa. Conforme a Figura 2, é possível perceber o ano de mais produção e a base de dados que mais contribuiu a esta pesquisa. 


\section{Figura 1- Frequência de artigos por ano e contribuição das bases de dados}

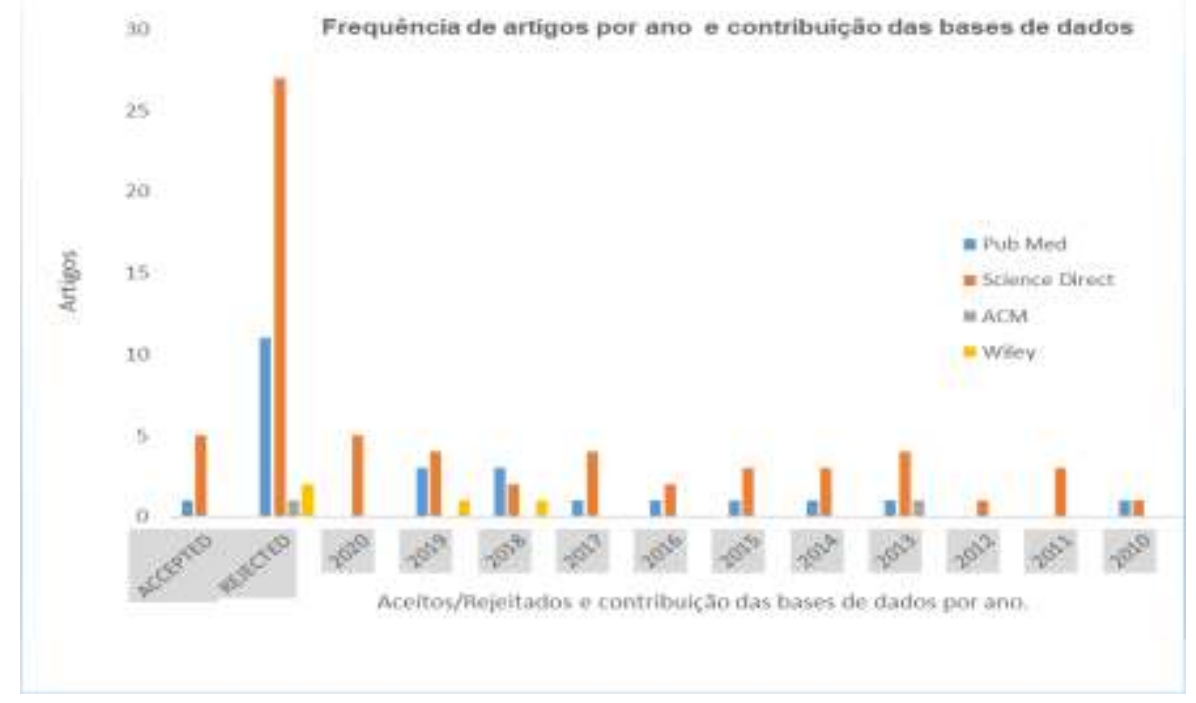

Fonte: Elaborado pelas autoras, com base nos dados da pesquisa (2020).

Observa-se que a base de dados que mais publicou o tema foi a Science Direct com $68 \%$ de publicações, o ano de maior publicação foi em 2020, porém neste período para esta pesquisa não houve contribuição. As publicações aceitas desta base de dados, para esta revisão sistemática da literatura, foram uma em 2010, uma em 2014, uma em 2015, uma em 2016 e uma em 2019.

A segunda base de dados que mais contribuiu a pesquisa foi a PubMed, com 25,53\% dos artigos tendo os anos de 2018 e 2019 de maior número de publicações, sendo que, o artigo aceito para esta revisão, teve sua publicação no ano de 2014. Nota-se um aumento progressivo de publicações sobre a temática nos últimos três anos, com concentração de trabalhos publicados nas bases da Science Direct e PubMed, validando a eficácia destas bases no que tange ao assunto: "Tecnologia digital; Enfermagem; Hospital; Educação Permanente; Engajamento e Saúde”. Na Wiley, validou-se 4,25\%, publicando artigos nos anos de 2018 e 2019, enquanto a ACM em 2013 contempla com um artigo, ambos foram rejeitados pelo CE4. No mesmo momento que a IEEE e SCOPUS não contemplaram com nenhum artigo a esta pesquisa.

Os artigos selecionados reportam-se ao uso de tecnologia digital síncrona (WhatsApp, fóruns) (Pimmer et al. (2019); Mathew (2014); Pata, Santos \& Burchert (2016)) e assíncrona (plataformas que disponibiliza conteúdo da área da saúde e afins) (Frenk et al (2010); Frisch et al. (2014); Murphy et al. (2015)). As tecnologias digitais citadas nestes artigos foram adotadas como meios auxiliares no aprendizado, neste contexto Frenk et al. (2010), e Pimmer et al. (2019) citam sobre estagnação de currículos e as lacunas do conhecimento, logo após a graduação. Vinculado a este contexto, Barlem et al. (2012) retratam no estudo intitulado como "Fragilidades, Fortalezas e Desafios na formação do enfermeiro", a percepção do enfermeiro quanto ao seu preparo profissional, a partir da formação conferida pela graduação. Os dados foram buscados por meio de entrevistas semiestruturadas a oito enfermeiras atuantes no atendimento terciário. Relatam ainda que foi identificado "contradições entre a formação teórico-prática e a práxis profissional". Frisch et al. (2014) também fala sobre estas inequidades, caracterizam tal acontecimento, como falha coletiva de compartilhar avanços de saúde.

Identifica-se, através da pesquisa, um interesse crescente pela temática, principalmente dos países desenvolvidos e com mais envolvimento com tecnologias. Infere-se que estes países estão investindo na educação permanente das equipes de enfermagem, utilizando da tecnologia digital para a aquisição e compartilhamento de conhecimento. Deste modo, entende-se que há um aumento do nível da qualidade do profissional e do serviço prestado. 
O incentivo a educação continuada favorece ao desenvolvimento do potencial, aprendendo no seu próprio ritmo e conforme as necessidades (Lilian Bacich (2016); Mathew (2014)). A comunicação aberta, em múltiplas redes, é um elemento essencial para uma aprendizagem significativa, pela possibilidade de acesso, troca e compatibilização de ideias (Bacich \& Moran, 2017). Firsch et. al. (2014) descrevem que o uso da plataforma InspireNet, contribuiu ao aumento do conhecimento, apoio a pesquisa informal, ao desenvolvimento profissional, a comunicação formal e informal, promovendo uma forma interativa de conectividade. Deste modo, infere-se que a aprendizagem ocorre dentro das relações pessoais e não apenas baseado no desenvolvimento do conhecimento.

Vários são os elementos relativos à motivação a educação contínua com as tecnologias digitais já citadas anteriormente: falta de enfermeiras (os) com experiência no setor atuante (MAthew, 2014)); padronização e nivelamento de conhecimento na equipe (Murphy et al., 2015), promoção da conexão sócio profissional (Pimmer et al. 2019). Estes trabalhos apresentam motivos que favorecem a qualidade do serviço prestado. Porém, a lacuna existente percebida são os motivos intrínsecos que levam o profissional a buscar a formação contínua. Bacich e Moran (2017) revelam que a motivação para aprender está relacionada àquilo que seja significativo.

Observa-se, através da leitura dos seis artigos selecionados, que não há mensuração do envolvimento dos colaboradores com as propostas estabelecidas pelas instituições, portanto, compreende-se que os colaboradores podem decidir o quanto se envolver com o que é oferecido. Isso pode ser relevante em países sem requisitos nacionais de desenvolvimento profissional. Por exemplo, na Holanda, conforme Pool et al. (2015) os enfermeiros para renovação do registro precisam demonstrar requisitos mínimos de prática. Já aqui no Brasil, Adamy et al. (2018) citam alguns desafios como a rotatividade nos setores e a infraestrutura adequada para o desenvolvimento entre o ensino-serviço.

\section{Conclusão}

Este trabalho analisou pesquisas referente ao uso de tecnologia digital na formação permanente do profissional de enfermagem no ambiente hospitalar. O mapeamento sistemático da literatura possibilitou identificar e analisar os artigos selecionados neste estudo.

Os artigos finais contemplam o objetivo desta revisão e exemplificaram algumas tecnologias digitais que estão sendo usadas pelas equipes de enfermagem como fomento a aprendizagem contínua. Percebe-se, através da leitura dos artigos selecionados, a influência que o conhecimento traz na práxis da equipe de enfermagem, apoderando o profissional, contribuindo para a mudança do ambiente e a melhora na qualidade de vida no trabalho.

A partir desta revisão da literatura, foi possível gerar embasamento necessário ao marco teórico da dissertação futura e identificar uma lacuna quanto a tecnologia digital na formação permanente em saúde. Neste sentido, percebe-se que o uso das tecnologias pode contribuir para o engajamento as equipes de enfermagem em ambientes hospitalares nos seus processos formativos.

Algumas limitações podem ser percebidas no desenvolvimento do trabalho. Apesar das tentativas de amenizar riscos, algumas escolhas podem afetar os resultados, como exemplo a escolha das bases de dados. Selecionou-se seis bases de dados, porém, algumas bases não contém o objetivo pretendido. O processo de busca, critérios utilizados como exclusão e inclusão também podem ter afetado o resultado, podendo ter excluído artigos pertinentes.

Com esta revisão, nota-se a falta de publicação de artigos nacionais referente as tecnologias digitais adotadas para auxílio na educação permanente aos profissionais da saúde. Podemos perceber, portanto, a baixa produtividade nacional desta temática. Podemos refletir que este elemento pode estar relacionado com a falta de motivação do profissional, cultura do ensino tradicional (sala de aula) ou ainda, a falta de incentivo da instituição a qual presta serviço. Analisar esses aspectos se 
constituem em um elemento importante de pesquisas futuras. Bem como, a possibilidade de nova análise, considerando os trabalhos publicados no restante de 2020 e no ano de 2021, visto que a pandemia pode ter influenciado quanto a aceleração do uso de tecnologias digitais em processo de formação.

\section{Agradecimentos}

O presente trabalho foi realizado com apoio da Coordenação de Aperfeiçoamento de Pessoal de Nível Superior Brasil (CAPES) - Código de Financiamento 001. Os autores também agradecem o apoio do Conselho Nacional de Desenvolvimento Científico e Tecnológico - CNPq.

\section{Referências}

Adamy, E. K., Zocche, D. A. de A., Vendruscolo, C., Metelski, F. K., Argenta, C., \& Valentini, J. D. S. (2018). Tecendo a educacao permanente em saúde no contexto hospitalar: relato de experinêcia. Revista de Enfermagem Do Centro-Oeste Mineiro, 8(0). https://doi.org/10.19175/recom.v8i0.1924

Almeida, R. G. dos S., Teston, E. F., \& Medeiros, A. de A. (2019). A interface entre o PET-Saúde/ Interprofissionalidade e a Política Nacional de Educação Permanente em Saúde. Saúde Em Debate, 43, 97-105.

Bacich, L., \& Moran, J. (2017). Metodologias ativas para uma educação inovadora uma abordagem teórica prática. (Penso, Ed.). Porto Alegre: recurso online.

Barlem, J. G. T., Lunardi, V. L., Barlem, E. L. D., Bordignon, S. S., Zacarias, C. C., \& Lunardi Filho, W. D. (2012). Fragilidades, fortalezas e desafios na formação do enfermeiro. Escola Anna Nery, 16(2), 347-363. https://doi.org/10.1590/s1414-81452012000200020

BES, P. (2017). Andragogia e educação profissional. (SER-SAGA). Porto Alegre: recursos online.

Brasil. Ministério da Saúde.Secretaria de gestão do trabalho e da educação na Saúde. Departamento de Gestão da educação na Saúde. (2018). Política Nacional de Educação Permanente em Saúde: o que se tem produzido para o seu fortalecimento? (MS, Ed.). Brasília. Retrieved from www.saude.gov.br/sgtes

Flores, G. E., Oliveira, D. L. L. de, \& Zocche, D. A. de A. (2016). Educação permanente no contexto hospitalar: a experiência que ressignifica o cuidado em enfermagem. Trabalho, Educação e Saúde, 14(2), 487-504. https://doi.org/10.1590/1981-7746-sip00118

Frenk, J., Chen, L., Bhutta, Z. A., Cohen, J., Crisp, N., Evans, T., ... Zurayk, H. (2010, December 4). Health professionals for a new century: Ttransforming education to strengthen health systems in an interdependent world. The Lancet. Lancet Publishing Group. https://doi.org/10.1016/S0140-6736(10)61854-5

Frisch, N., Atherton, P., Borycki, E., Mickelson, G., Cordeiro, J., Lauscher, H. N., \& Black, A. (2014). Growing a professional network to over 3000 members in less than 4 years: Evaluation of InspireNet, British Columbia's virtual nursing health services research network. Journal of Medical Internet Research, 16(2), e49. https://doi.org/10.2196/jmir.3018

Marquis, B. L., \& Huston, C. J. (2005). Administração e Liderança em Enfermagem. (Artmed, Ed.) (4 .ed.). Porto Alegre.

Mathew, B. (2014). Using a social networking tool for blended learning in staff training: Sharing experience from practice. Journal of Neonatal Nursing, 20(3), 90-94. https://doi.org/10.1016/j.jnn.2014.03.005

Munhoz, A. S. (2017). Andragogia: a educação de jovens e de adultos em ambientes virtuais. (Intersaberes, Ed.). Curitiba, PR.

Murphy, J., Worswick, L., Pulman, A., Ford, G., \& Jeffery, J. (2015). Translating research into practice: Evaluation of an e-learning resource for health care professionals to provide nutrition advice and support for cancer survivors. Nurse Education Today, 35(1), 271-276. https://doi.org/10.1016/j.nedt.2014.05.009

Paschoal, A. S., Mantovani, M. de F., \& Méier, M. J. (2007). Percepçao da educaçao permanente, continuada e em serviço para enfermeiros de um hospital de ensino. Revista Da Escola de Enfermagem, 41(3), 478-484. https://doi.org/10.1590/s0080-62342007000300019

Pata, K., Santos, P., \& Burchert, J. (2016). Social recognition provision patterns in professional Q\&A forums in Healthcare and Construction. Computers in Human Behavior, 55, 571-583. https://doi.org/10.1016/j.chb.2015.06.046

Petersen, K., Vakkalanka, S., \& Kuzniarz, L. (2015). Guidelines for conducting systematic mapping studies in software engineering: An update. In Information and Software Technology (Vol. 64, pp. 1-18). Elsevier. https://doi.org/10.1016/j.infsof.2015.03.007

Pimmer, C., Brühlmann, F., Odetola, T. D., Oluwasola, D. O., Dipeolu, O., \& Ajuwon, A. J. (2019). Facilitating professional mobile learning communities with instant messaging. Computers and Education, 128, 102-112. https://doi.org/10.1016/j.compedu.2018.09.005

Pool, I. A., Poell, R. F., Berings, M. G. M. C., \& Ten Cate, O. (2015). Strategies for continuing professional development among younger, middle-aged, and older nurses: A biographical approach. International Journal of Nursing Studies, 52(5), 939-950. https://doi.org/10.1016/j.ijnurstu.2015.02.004

Spader, G. (2019). A influência da competência profissional, da inteligência emocional, da produtividade e do engajamento sobre a satisfação com o trabalho em um ambiente de serviços de saúde. Universidade de Caxias do Sul.

Wunsch, G., Righi, R. da R., Costa, C. A. da, \& Oliveira, K. F. de. (2018). A última década do uso de dispositivos da internet das coisas durante a triagem de pacientes no Departamento de Emergência-um Mapeamento Sistemático. ... of Health Informatics, 10(3), 95-100. http://www.jhi-sbis.saude.ws/ojsjhi/index.php/jhi-sbis/article/view/564 